\title{
Deviation of polynomials from their expectations and isoperimetry
}

\author{
LAVRENTIN M. ARUTYUNYAN* and EGOR D. KOSOV** \\ National Research University Higher School of Economics, Moscow, Russia. \\ E-mail: *lavrentin@yandex.ru; ${ }^{* *}$ ked_2006@mail.ru
}

The article is divided into two parts. In the first part, we study the deviation of a polynomial from its mathematical expectation. This deviation can be estimated from above by Carbery-Wright inequality, so we investigate estimates of the deviation from below. We obtain such type estimates in two different cases: for Gaussian measures and a polynomial of an arbitrary degree and for an arbitrary log-concave measure but only for polynomials of the second degree. In the second part, we deal with the isoperimetric inequality and the Poincaré inequality for probability measures on the real line that are images of the uniform distributions on convex compact sets in $\mathbb{R}^{n}$ under polynomial mappings.

Keywords: Carbery-Wright inequality; distribution of a polynomial; Gaussian measure; isoperimetric inequality; logarithmically concave measure; Poincaré inequality

Polynomials on spaces endowed with log-concave measures possess a number of important and useful properties. These properties have been studied in many works, see, for example, $[1,4,5$, $16,26]$. Some authors (see $[11,12,27,28]$ ) also consider the special case of Gaussian measures.

Results about polynomial images of log-concave measures find many applications in various fields. One of such applications is concerned with geometrical properties of convex bodies, especially when the dimension tends to infinity. For example, Bourgain [16] proved an upper bound in the hyperplane conjecture using a Khinchin-type inequality for polynomials of a fixed degree on convex bodies (about this inequality see also [4,5]). It should be remarked that the best known upper bound in the hyperplane conjecture is due to Klartag (see [23]). On the other hand, properties of polynomials and polynomial distributions play an important role in probabilistic questions. In particular, Gaussian measures are also logarithmically concave and there are certain properties of Gaussian measures that were proved only in the framework of general logarithmically concave measures. One of them is the following Carbery-Wright inequality that holds for any polynomial $f$ of degree $d$ and any log-concave measure $\mu$ on $\mathbb{R}^{n}$ (see [17], Theorem 2):

$$
\|f\|_{L^{1}(\mu)}^{1 / d} \mu(x:|f(x)| \leq \alpha) \leq C(d) \alpha^{1 / d} .
$$

This inequality has already found interesting applications in probability theory (see, for example, $[27,28])$. Concentration and anti-concentration inequalities play an important role in modern probability theory. One of such inequalities is well-known estimate of large deviation for Lipschitz functions on Gaussian space (see [9], Theorem 4.5.6). On the other hand, many articles study Lévy concentration function (see [20,24]), which is closely related to the anti-concentration inequalities, and which is used in study of properties of random matrices (see $[29,30])$. 
In the first part of this work, we discuss inequalities that are reverse in some sense to the Carbery-Wright inequality, more precisely, inequalities of the form

$$
\mu\left(\left|f-m_{f}\right| \leq \sigma_{f} s\right) \geq C(d) \varphi(s),
$$

where $f$ is a polynomial of degree $d$ and $m_{f}$ and $\sigma_{f}^{2}$ are its expectation and variance, respectively, and $s \in[0,1 / 2]$. We prove inequalities of such a type in two different cases. In the first case, the measure $\mu$ is Gaussian and $\varphi(s)=s|\ln s|^{-d / 2}$ (see Theorem 2.3). In the second case, the measure $\mu$ is an arbitrary log-concave measure and the degree of the polynomial $f$ is at most two while $\varphi(s)=s$ (see Theorem 2.9). The main feature of our inequalities is their independence of the dimension of the space and of the measure $\mu$ itself.

The second part of our work is devoted to the isoperimetric inequality and the Poincare inequality for distributions of polynomials. It is well known that for the standard Gaussian measure on $\mathbb{R}^{n}$ both inequalities hold true (see $[9,15,31]$ ). For log-concave measures, inequalities of these types have been studied in $[3,22]$. In our work, these inequalities are proved for probability measures on the real line that are polynomial images of the uniform distributions on convex compact sets in $\mathbb{R}^{n}$ (see Theorem 3.5 and Corollaries 3.6 and 3.7). The study of the Poincaré inequality for the distributions of random variables is an important step in the study of the Poincare inequality for multidimensional measures (see [6]). For example, it is known that the Poincare inequality is stable under taking product of measures (see [7,8]). The work [8] established the precise value of constant in the isoperimetric inequality and in the Poincaré inequality for one dimensional distributions, but it is given in terms of the distribution function and the density of the distribution that are usually hard to calculate explicitly. In our case, we obtain the estimate of these constants by other means, which allows to avoid difficulties concerning study of the distribution function.

One of the main tools used in this paper is the so-called localization technique (see [22,25]). The idea of localization of a problem was used in many papers as an approach for obtaining estimates in multidimensional spaces. For example, it was used to study isoperimetric inequalities for the uniform distributions on convex bodies in [22] and for the distributions on spheres in [21]. Also it was used in [4,5] in the proof of Khinchin-type inequalities for polynomials. This technique allows to reduce some multidimensional inequalities to one-dimensional ones. In the case of polynomials it is especially convenient, because a restriction of a polynomial to a straight line is again a polynomial. A new approach to the ideas of localization was developed in [19], where localization is interpreted as a property of extreme points of some special convex sets in the space of all probability measures.

\section{Preliminaries}

First of all, we introduce some notation and mention certain previously known results used in our work.

Let $\mu$ be a probability measure on $\mathbb{R}^{n}$ and let $f$ be a $\mu$-measurable function. We use the following notation:

$$
\mu_{f}=\mu \circ f^{-1} \quad \text { is the image of the measure } \mu \text { under the mapping } f,
$$




$$
\begin{aligned}
m_{f} & =\int f d \mu \quad \text { is the expectation of the random variable } f, \\
\sigma_{f}^{2} & =\int\left(f-m_{f}\right)^{2} d \mu \quad \text { is the variance of the random variable } f, \\
\alpha_{f} & =\int\left|f-m_{f}\right| d \mu, \\
\|f\|_{p} & =\left(\int|f|^{p} d \mu\right)^{1 / p} \quad \text { for } p>0, \quad\|f\|_{0}=\exp \left(\int \ln |f| d \mu\right)=\lim _{r \rightarrow 0}\|f\|_{r} .
\end{aligned}
$$

Let $I_{A}$ denote the indicator function of a set $A$.

A probability measure $\mu$ on $\mathbb{R}^{n}$ is called logarithmically concave (also log-concave or convex) if it has a density of the form $e^{-V}$ with respect to Lebesgue measure on some affine subspace, where $V$ is a convex function (possibly with infinite values) on this subspace (see [10]). This property is equivalent (see $[13,14]$ ) to the property that for every pair of Borel sets $A, B$ one has

$$
\mu(t A+(1-t) B) \geq \mu(A)^{t} \mu(B)^{1-t}, \quad \forall t \in[0,1] .
$$

There are two important examples of log-concave measures. First, a uniform distribution on an arbitrary convex body in $\mathbb{R}^{n}$ is a logarithmically concave measure. Second, any Gaussian measure (e.g., the Wiener measure generated by the Wiener process) is also a log-concave measure.

A polynomial of degree $d$ is a function $f$ on $\mathbb{R}^{n}$ of the form

$$
f(x)=\sum_{m=0}^{d} B_{m}(x, \ldots, x)=\sum_{m=0}^{d} \sum_{i_{i}+\cdots+i_{n}=m} b_{i_{1}, \ldots, i_{n}}^{m} x_{1}^{i_{1}} \cdots x_{n}^{i_{n}},
$$

where $B\left(x_{1}, \ldots, x_{m}\right)$ is a symmetric $m$-linear function.

Let $v$ be a probability measure on the real line. Define the $v$-perimeter of a set $A$ by the following formula:

$$
v^{+}(A)=\liminf _{\varepsilon \rightarrow 0} \frac{v(A+(-\varepsilon, \varepsilon))-v(A)}{\varepsilon} .
$$

The proofs of our main results use the following known facts.

Theorem 1.1 (see [4], Theorem 1, [5], Theorem 3). There is an absolute constant c such that, for every log-concave measure $\mu$ on $\mathbb{R}^{n}$, every polynomial $f$ of degree $d$, and every number $q \geq 1$, the following inequalities hold true:

$$
\|f\|_{q} \leq(c q d)^{d}\|f\|_{0}, \quad\|f\|_{q} \leq(c q)^{d}\|f\|_{1} .
$$

The property in the theorem above is sometimes called hypercontractivity, especially when we deals with Gaussian measures, when polynomials are eigenfunctions of the Ornstein-Uhlenbeck operator.

Some infinite-dimensional analogues of previous theorem are presented in [1]. 
Theorem 1.2 (see [1], Theorem 2.1). There is an absolute constant $C$ such that, for every logconcave measure $\mu$ on $\mathbb{R}^{n}$, every set $U$ of positive $\mu$-measure, and every polynomial $f$ of degree $d$, the following estimate holds:

$$
\mu(U)^{d+1} \int|f| d \mu \leq(C d)^{2 d} \int_{U}|f| d \mu
$$

Let us recall two localization lemmas. The first one is concerned with log-concave measures.

Theorem 1.3 (see [19], Theorem 1 and Corollary 1, [22], Theorem 2.7). Let $f_{1}, f_{2}$ be a pair of two upper semi-continuous nonnegative functions on $\mathbb{R}^{n}$ and let $f_{3}, f_{4}$ be a pair of two lower semi-continuous nonnegative functions on $\mathbb{R}^{n}$. Suppose that for every compact interval $\Delta=$ $[a, b] \subset \mathbb{R}^{n}$ and every measure $v$ with a density of the form $e^{\ell}$ with respect to Lebesgue measure on $\Delta$, where $\ell$ is an affine function on $\Delta$, one has

$$
\left(\int_{\Delta} f_{1} d v\right)^{\alpha}\left(\int_{\Delta} f_{2} d v\right)^{\beta} \leq\left(\int_{\Delta} f_{3} d v\right)^{\alpha}\left(\int_{\Delta} f_{4} d v\right)^{\beta} .
$$

Then the following inequality holds for every log-concave measure $\mu$ on $\mathbb{R}^{n}$ :

$$
\left(\int f_{1} d \mu\right)^{\alpha}\left(\int f_{2} d \mu\right)^{\beta} \leq\left(\int f_{3} d \mu\right)^{\alpha}\left(\int f_{4} d \mu\right)^{\beta} .
$$

The second localization lemma is applicable in the case of uniform distributions on convex bodies.

Theorem 1.4 (see [19], Theorem 1 and Corollary 1, [22], Corollary 2.2, [25], Lemma 2.5). Let $f_{1}, f_{2}$ be a pair of two upper semi-continuous nonnegative functions on $\mathbb{R}^{n}$ and let $f_{3}, f_{4}$ be a pair of two lower semi-continuous nonnegative functions on $\mathbb{R}^{n}$. Suppose that for every compact interval $\Delta=[a, b] \subset \mathbb{R}^{n}$ and every measure $v$ with a density of the form $(\alpha t+\beta)^{n-1}$ with respect to Lebesgue measure on $\Delta$ one has

$$
\left(\int_{\Delta} f_{1} d v\right)^{\alpha}\left(\int_{\Delta} f_{2} d v\right)^{\beta} \leq\left(\int_{\Delta} f_{3} d v\right)^{\alpha}\left(\int_{\Delta} f_{4} d v\right)^{\beta} .
$$

Then the following inequality holds for every convex body $K$ in $\mathbb{R}^{n}$ :

$$
\left(\int_{K} f_{1} d \lambda\right)^{\alpha}\left(\int_{K} f_{2} d \lambda\right)^{\beta} \leq\left(\int_{K} f_{3} d \lambda\right)^{\alpha}\left(\int_{K} f_{4} d \lambda\right)^{\beta},
$$

where $\lambda$ is Lebesgue measure. 


\section{Behavior of the distribution of a polynomial in a neighbourhood of its expectation}

In this section, we estimate from below the measure of small deviations of a polynomial from its mean.

In the following lemma, we present the proof, kindly proposed by one of our referees, which is far shorter than the original one.

Lemma 2.1. For every number $d \in \mathbb{N}$ there are positive constants $c(d)$ and $s(d)$ depending only on the number $d$ such that for every log-concave measure $\mu$ on $\mathbb{R}^{n}$ and for every polynomial $f$ of degree $d$ with $\alpha_{f}>0$ and $m_{f}=0$ one has

$$
\mu\left(f \geq \varepsilon \alpha_{f}\right) \geq c(d) \quad \text { for every number } \varepsilon \in[0, s(d)] .
$$

Proof. Note that in our case $\|f\|_{1}=\alpha_{f}$, since the polynomial $f$ is of zero mean. By Theorem 1.1 and by Carbery-Wright inequality ([17], Theorem 2), one has

$$
\begin{aligned}
\alpha_{f} & =\int|f| d \mu=\int_{f>0} f d \mu-\int_{f<0} f d \mu=2 \int_{f>0} f d \mu \\
& =2\left(\int_{f \geq \varepsilon \alpha_{f}} f d \mu+\int_{0<f<\varepsilon \alpha_{f}} f d \mu\right) \leq 2\|f\|_{2}\left(\sqrt{\mu\left(f \geq \varepsilon \alpha_{f}\right)}+\sqrt{\mu\left(|f|<\varepsilon \alpha_{f}\right)}\right) \\
& \leq 2(2 c)^{d} \alpha_{f}\left(\sqrt{\mu\left(f \geq \varepsilon \alpha_{f}\right)}+\sqrt{C(d) \varepsilon^{1 / d}}\right),
\end{aligned}
$$

where in the third equality we have used the fact that $m_{f}=0$. Thus, we have

$$
\mu\left(f \geq \varepsilon \alpha_{f}\right) \geq\left(2^{-1}(2 c)^{-d}-\sqrt{C(d) \varepsilon^{1 / d}}\right)^{2} .
$$

We immediately conclude the statement of the lemma taking $\varepsilon$ small enough.

Taking $\varepsilon=0$ one can obtain the following corollary.

Corollary 2.2. For every number $d \in \mathbb{N}$ there is a constant $c(d) \in(0,1)$ depending only on $d$ such that for every log-concave measure $\mu$ on $\mathbb{R}^{n}$ and for every polynomial $f$ of degree $d$ with $\sigma_{f}>0$ one has

$$
1-c(d) \geq \mu\left(f<m_{f}\right) \geq c(d) .
$$

\section{A. The case of a Gaussian measure}

First, we consider the case of the standard Gaussian measure on $\mathbb{R}^{n}$ and a polynomial of an arbitrary degree.

Below we need the following elementary estimate. Let $f$ be a smooth function on $\mathbb{R}^{n}$. Let $D^{m} f(x)$ denote the $m$-fold derivative of $f$, that is, a multilinear function such that

$$
\left.\frac{d^{m}}{d t^{m}} f(x+t h)\right|_{t=0}=D^{m} f(x)(h, \ldots, h) .
$$


Let now $f$ be a polynomial of degree $d$ on $\mathbb{R}^{n}$. Set

$$
\varphi(t)=f(x+t(y-x)) .
$$

Then one has

$$
f(y)=\varphi(1)=\varphi(0)+\sum_{m=1}^{d}(m !)^{-1} \varphi^{(m)}(0)=f(x)+\sum_{m=1}^{d}(m !)^{-1} D^{m} f(x)(y-x, \ldots, y-x),
$$

thus,

$$
|f(y)-f(x)| \leq \sum_{m=1}^{d}(m !)^{-1}\left|D^{m} f(x)\right||y-x|^{m},
$$

where $\left|D^{m} f(x)\right|^{2}=\sum_{i_{1}, \ldots, i_{m}}\left|\partial_{x_{i_{1}}, \ldots, x_{i_{m}}} f(x)\right|^{2}$.

We also need the following estimate for the standard Gaussian measure $\gamma$ on $\mathbb{R}^{n}$ :

$$
\left\|D^{m} f\right\|_{2}^{2}:=\int\left|D^{m} f\right|^{2} d \gamma \leq a(m, d) \sigma_{f}^{2},
$$

where the constant $a(m, d)$ depends only on $m$ and $d$ and is independent of the dimension $n$. This estimate follows from the equivalence of the Sobolev and $L^{p}$-norms on the space of all polynomials of a fixed degree (see [9], Corollary 5.5.5).

Let us recall the isoperimetric inequality for Gaussian measures (see [31], Theorem 3 and Theorem 4, [15], Theorem 3.1, [9], Theorem 4.3.1), which is used in the proof of the next theorem. Let $\gamma$ be the standard Gaussian measure on $\mathbb{R}^{n}$, let $C$ be a measurable set in $\mathbb{R}^{n}$, and let $B$ be the closed unit ball centered at the origin. Then

$$
\gamma(C+\varepsilon B) \geq \Phi(a+\varepsilon)
$$

where $\Phi$ is the distribution function of the standard Gaussian random variable on the real line and the number $a$ is chosen from the relation $\gamma(C)=\Phi(a)$.

For a Gaussian measure, there is the following estimate for the tails of a polynomial $f$ of degree $d$ :

$$
\gamma\left(|f|>\|f\|_{2} t^{d}\right) \leq R \exp \left(-r t^{2}\right)
$$

for every $t>0$ and some positive constants $R$ and $r$ (see [9], Corollary 5.5.7).

Theorem 2.3. Let $\gamma$ be the standard Gaussian measure on $\mathbb{R}^{n}$. Then for every number $d \in \mathbb{N}$ there exists a number $L(d)>0$, which depends only on $d$ and is independent of anything else, such that for every polynomial $f$ of degree $d$ the estimate

$$
\gamma\left(\left|f-m_{f}\right| \leq \sigma_{f} s\right) \geq L(d) s|\ln s|^{-d / 2} \quad \text { holds for every } s \in(0,1 / 2] .
$$


Proof. Without loss of generality, we can assume that $m_{f}=0$. Fix a number $t>1$ (this number will be chosen later). Let $B$ be the unit ball in $\mathbb{R}^{n}$ centered at the origin. For $\varepsilon<1$ consider the set

$$
A=(\{f<0\}+\varepsilon B) \cap(\{f>0\}+\varepsilon B) \cap \bigcap_{m=1}^{d}\left\{\left|D^{m} f(x)\right| \leq t^{d-m}\left\|D^{m} f\right\|_{2}\right\} .
$$

Note that by (2.1) we have $A \subset\left\{|f| \leq \sum_{m=1}^{d}(m !)^{-1} t^{d-m}\left\|D^{m} f\right\|_{2} \varepsilon^{m}\right\}$. Since $t>1$ and $\varepsilon<1$, we have $\varepsilon^{m} \leq \varepsilon, t^{d-m} \leq t^{d}$, and inequality (2.2) yields the estimate

$$
\sum_{m=1}^{d}(m !)^{-1} t^{d-m}\left\|D^{m} f\right\|_{2} \varepsilon^{m} \leq C(d) \sigma_{f} t^{d} \varepsilon
$$

where $C(d)$ is a constant, which depends only on the degree $d$ of a polynomial. Thus, we have the inclusion

$$
A \subset\left\{|f| \leq C(d) \sigma_{f} t^{d} \varepsilon\right\} .
$$

Let $a_{f}$ be the number such that $\gamma(f<0)=\Phi\left(a_{f}\right)$. Using the Gaussian isoperimetric inequality (2.3), we can estimate the measure of the set $A$ as follows:

$$
\begin{aligned}
\gamma(A) \geq & 1-(1-\gamma(\{f<0\}+\varepsilon B))-(1-\gamma(\{f>0\}+\varepsilon B)) \\
& -\sum_{m=1}^{d} \gamma\left(\left|D^{m} f(x)\right| \geq t^{d-m}\left\|D^{m} f\right\|_{2}\right) \\
\geq & -1+\Phi\left(a_{f}+\varepsilon\right)+\Phi\left(-a_{f}+\varepsilon\right)-R \sum_{m=1}^{d} e^{-r t^{2}} \\
= & \Phi\left(a_{f}+\varepsilon\right)-\Phi\left(a_{f}\right)+\Phi\left(-a_{f}+\varepsilon\right)-\Phi\left(-a_{f}\right)-R d e^{-r t^{2}}
\end{aligned}
$$

In the second inequality, estimate (2.4) and isoperimetric inequality (2.3) were used and in the last step the equality $\Phi\left(-a_{f}\right)=1-\Phi\left(a_{f}\right)$ was used.

By Corollary 2.2, there is a number $c(d)$ such that

$$
1-c(d) \geq \gamma\left(f<m_{f}\right) \geq c(d),
$$

so, there is a constant $a(d)$ such that for every polynomial $f$ of degree $d$ with zero expectation one has $\left|a_{f}\right| \leq a(d)$. Let $q(d)$ be the minimal value of the standard Gaussian density on the interval $[-a(d)-1, a(d)+1]$. Then $\Phi\left(a_{f}+\varepsilon\right)-\Phi\left(a_{f}\right) \geq q(d) \varepsilon$ and similarly $\Phi\left(-a_{f}+\varepsilon\right)-$ $\Phi\left(-a_{f}\right) \geq q(d) \varepsilon$. Therefore,

$$
\gamma\left(|f| \leq C(d) \sigma_{f} t^{d} \varepsilon\right) \geq \gamma(A) \geq 2 q(d) \varepsilon-R d e^{-r t^{2}} .
$$


Let $0<s<e^{-1}, \varepsilon=C(d)^{-1} 2^{-d} r^{d / 2} s|\ln s|^{-d / 2}, t=2 r^{-1 / 2}|\ln s|^{1 / 2}$. Then

$$
\begin{aligned}
\gamma\left(|f| \leq \sigma_{f} s\right) & \geq 2 q(d) C(d)^{-1} 2^{-d} r^{d / 2} s|\ln s|^{-d / 2}-R d e^{-2|\ln s|} \\
& =2 q(d) C(d)^{-1} 2^{-d} r^{d / 2} s|\ln s|^{-d / 2}-R d s^{2} .
\end{aligned}
$$

There is a number $s_{0}=s_{0}(d) \in\left(0, e^{-1}\right)$ such that for any $s \leq s_{0}$ one has

$$
\gamma\left(|f| \leq \sigma_{f} s\right) \geq q(d) C(d)^{-1} 2^{-d} r^{d / 2} s|\ln s|^{-d / 2} .
$$

For every $s \in\left(s_{0}, 1 / 2\right]$, we have

$$
\begin{aligned}
\gamma\left(|f| \leq \sigma_{f} s\right) & \geq \gamma\left(|f| \leq \sigma_{f} s_{0}\right) \geq q(d) C(d)^{-1} 2^{-d} r^{d / 2} s_{0}\left|\ln s_{0}\right|^{-d / 2} \\
& \geq q(d) C(d)^{-1} 2^{-d} r^{d / 2} s_{0}\left|\ln s_{0}\right|^{-d / 2}\left(\max _{\tau \in\left[s_{0}, 1 / 2\right]} \tau|\ln \tau|^{-d / 2}\right)^{-1} s|\ln s|^{-d / 2} .
\end{aligned}
$$

Thus, there is a positive constant $L(d)$ such that

$$
\gamma\left(|f| \leq \sigma_{f} s\right) \geq L(d) s|\ln s|^{-d / 2},
$$

whenever $0 \leq s \leq 1 / 2$.

Remark 2.4. We note that up to a logarithmic factor $|\ln s|^{-d / 2}$, the estimate in the previous theorem is close to optimal. Indeed, one can find a polynomial $f$ such that $f(x)=\left(x-m_{f}\right)\left(x^{d-1}+\right.$ $\left.x^{d-2}\right)$. For such a polynomial we have $f(x) \sim C\left(x-m_{f}\right)$ in the neighborhood of the point $m_{f}$, so, $\gamma(|f| \leq s) \sim C_{1} s$ for small $s$.

\section{B. The case of a log-concave measure and a polynomial of degree two}

Here we obtain an estimate that is sharper than the previous one in the case of an arbitrary log-concave measure, but only for polynomials of degree 2 . The proof in this case relies on the following lemma.

Lemma 2.5. There is an absolute constant c such that for every polynomial $f$ of degree two on the real line and for every pair of positive numbers $s$ and $\varepsilon$ we have the inequality

$$
\varepsilon \int_{0}^{s} e^{-t} I_{\{f \leq-\varepsilon\}} d t \int_{0}^{s} e^{-t} I_{\{f \geq \varepsilon\}} d t \leq c \int_{0}^{s} e^{-t} I_{\{|f|<\varepsilon\}} d t \int_{0}^{s} e^{-t}|f(t)| d t .
$$

Proof. Without loss of generality, we can assume that the coefficient at the highest degree term of the polynomial $f$ is 1 . Suppose first that $s \geq 1$.

If the left-hand side is not zero, then there is a root of the polynomial $f$ in the interval $(0, s)$. Let $\tau$ denote the minimal root in $(0, s)$ and let $\sigma$ be the second root (which may or may not be in this interval). Then on the interval $(0, \tau)$ the polynomial $f$ is either strictly positive or strictly negative, hence,

$$
\int_{0}^{s} e^{-t} I_{\{f \leq-\varepsilon\}} d t \int_{0}^{s} e^{-t} I_{\{f \geq \varepsilon\}} d t \leq \int_{\tau}^{\infty} e^{-t} d t=e^{-\tau}
$$


Thus, we have to obtain the estimate

$$
\varepsilon e^{-\tau} \leq c \int_{0}^{s} e^{-t} I_{\{|f|<\varepsilon\}} d t \int_{0}^{s} e^{-t}|f(t)| d t .
$$

By Theorems 1.1 and 1.2 for a polynomial $g$ of degree $d$, affine functions $\ell_{1}, \ell_{2}$ and a logconcave measure $v$ we have

$$
\begin{aligned}
\|g\|_{L^{1}(v)} & \geq(2 c)^{-d}\|g\|_{L^{2}(v)}, \\
16 c^{2}\left\|\ell_{1}\right\|_{L^{2}(v)}^{2}\left\|\ell_{2}\right\|_{L^{2}(v)}^{2} & \geq\left\|\ell_{1} \ell_{2}\right\|_{L^{2}(v)}^{2} \geq(2 c)^{-2}\left\|\ell_{1}\right\|_{L^{2}(v)}^{2}\left\|\ell_{2}\right\|_{L^{2}(v)}^{2}, \\
\int_{A}|g| d v & \geq(C d)^{-2 d} v(A)^{1+d} \int|g| d v,
\end{aligned}
$$

where in the second estimate for the right-hand side we used the inequality $\left\|\ell_{1} \ell_{2}\right\|_{2} \geq\left\|\ell_{1} \ell_{2}\right\|_{0}$ and the multiplicative property of the $\|\cdot\|_{0}\left(\left\|\ell_{1} \ell_{2}\right\|_{0}=\left\|\ell_{1}\right\|_{0}\left\|\ell_{2}\right\|_{0}\right)$ along with Theorem 1.1 and for the left-hand side we used Theorem 1.1, the multiplicative property of the $\|\cdot\|_{0}$, and the estimates $\left\|\ell_{1}\right\|_{0} \leq\left\|\ell_{1}\right\|_{2},\left\|\ell_{2}\right\|_{0} \leq\left\|\ell_{2}\right\|_{2}$. Applying these inequalities to the functions $g=f$, $\ell_{1}(t)=t-\tau, \ell_{2}(t)=t-\sigma$ and to the measure $d v=e^{-t} I_{\{t>0\}} d t$, we obtain that

$$
\begin{aligned}
\int_{0}^{s} e^{-t}|f(t)| d t & \geq(2 C)^{-4}\left(1-e^{-s}\right)^{3} \int_{0}^{\infty} e^{-t}|f(t)| d t \\
& \geq(2 C)^{-4}(2 c)^{-2}(2 c)^{-2}\left(1-e^{-s}\right)^{3}\left(\int_{0}^{\infty}(t-\sigma)^{2} e^{-t} d t \int_{0}^{\infty}(t-\tau)^{2} e^{-t} d t\right)^{1 / 2} \\
& \geq(2 C)^{-4}(2 c)^{-2}(2 c)^{-2}\left(1-e^{-1}\right)^{3}\left(1+(1-\tau)^{2}\right)^{1 / 2}\left(1+(1-\sigma)^{2}\right)^{1 / 2}
\end{aligned}
$$

where in the last estimate we have used the inequality $1-e^{-s}>1-e^{-1}$ for $s \geq 1$ and we have calculated the integrals. Let us pick a point $u$ such that $\tau \in[u, u+1] \subset[0, s]$. Then $|t-\sigma| \leq$ $|\tau-\sigma|+1$ for $t \in[u, u+1]$.

Let us firstly consider the case $\varepsilon<\frac{1}{2}(1+|\tau-\sigma|)$. In this case

$$
\int_{0}^{s} e^{-t} I_{\{|f|<\varepsilon\}} d t \geq e^{-\tau-1} \int_{u}^{u+1} I_{\left\{|t-\tau|<\varepsilon(|\tau-\sigma|+1)^{-1}\right\}} d t \geq e^{-\tau-1} \varepsilon(|\tau-\sigma|+1)^{-1} .
$$

Note that

$$
\inf _{\tau, \sigma} \frac{\left(1+(1-\tau)^{2}\right)^{1 / 2}\left(1+(1-\sigma)^{2}\right)^{1 / 2}}{|\tau-\sigma|+1} \geq(\sqrt{3})^{-1}>0 .
$$

Thus, in the case when $\varepsilon<\frac{1}{2}(1+|\tau-\sigma|)$ the desired estimate is proved.

Now let us consider the case $\varepsilon \geq \frac{1}{2}(1+|\tau-\sigma|)$. If

$$
\varepsilon \leq 2\left(1-e^{-1}\right)^{-1} \int_{0}^{\infty} e^{-t}|f(t)| d t
$$


then

$$
\begin{aligned}
\int_{0}^{s} e^{-t} I_{\{|f|<\varepsilon\}} d t & \geq e^{-\tau-1} \int_{u}^{u+1} I_{\left\{|t-\tau|<\varepsilon(|\tau-\sigma|+1)^{-1}\right\}} d t \\
& \geq e^{-\tau-1} \int_{u}^{u+1} I_{\{|t-\tau|<1 / 2\}} d t \geq e^{-\tau-1} / 2 .
\end{aligned}
$$

Using the estimate

$$
\int_{0}^{s} e^{-t}|f(t)| d t \geq(2 C)^{-4}\left(1-e^{-1}\right)^{-1} \int_{0}^{\infty} e^{-t}|f(t)| d t,
$$

which was obtained above, we also get the desired inequality in this case. If now

$$
\varepsilon \geq 2\left(1-e^{-1}\right)^{-1} \int_{0}^{\infty} e^{-t}|f(t)| d t
$$

then, by Chebyshev's inequality

$$
\int_{0}^{s} e^{-t} I_{\{|f| \geq \varepsilon\}} d t \leq \int_{0}^{\infty} e^{-t} I_{\{|f| \geq \varepsilon\}} d t \leq \varepsilon^{-1} \int_{0}^{\infty} e^{-t}|f(t)| d t .
$$

On the other hand, using previous estimate and the fact, that $s \geq 1$, we have

$$
\begin{aligned}
\int_{0}^{s} e^{-t} I_{\{|f|<\varepsilon\}} d t & \geq \int_{0}^{s} e^{-t} d t-\int_{0}^{s} e^{-t} I_{\{|f| \geq \varepsilon\}} d t \\
& \geq 1-e^{-1}-\varepsilon^{-1} \int_{0}^{\infty} e^{-t}|f(t)| d t \geq\left(1-e^{-1}\right) 2^{-1}
\end{aligned}
$$

Using again the estimate (2.6) and estimating one of the integrals in the left-hand side of (2.5) by one, we have

$$
\begin{aligned}
\varepsilon \int_{0}^{s} e^{-t} I_{\{f \leq-\varepsilon\}} d t \int_{0}^{s} e^{-t} I_{\{f \geq \varepsilon\}} d t & \leq \varepsilon \int_{0}^{s} e^{-t} I_{\{|f| \geq \varepsilon\}} d t \leq \int_{0}^{\infty} e^{-t}|f(t)| d t \\
& \leq(2 C)^{4}\left(1-e^{-1}\right) \int_{0}^{s} e^{-t}|f(t)| d t \\
& \leq 2(2 C)^{4} \int_{0}^{s} e^{-t} I_{\{|f|<\varepsilon\}} d t \int_{0}^{s} e^{-t}|f(t)| d t
\end{aligned}
$$

Thus, the desired estimate is also valid in the considered case.

Now let $s<1$. In this case $e^{-1} \leq e^{-t} \leq 1$ on $[0, s]$ and our estimate in this case is equivalent to the following one:

$$
\varepsilon \int_{0}^{s} I_{\{f \leq-\varepsilon\}} d t \int_{0}^{s} I_{\{f \geq \varepsilon\}} d t \leq c \int_{0}^{s} I_{\{|f|<\varepsilon\}} d t \int_{0}^{s}|f(t)| d t .
$$


Moreover, after a linear change of variables, we can assume $s=1$, i.e., the desired inequality takes the form

$$
\varepsilon \int_{0}^{1} I_{\{f \leq-\varepsilon\}} d t \int_{0}^{1} I_{\{f \geq \varepsilon\}} d t \leq c \int_{0}^{1} I_{\{|f|<\varepsilon\}} d t \int_{0}^{1}|f(t)| d t,
$$

probably for some other $\varepsilon>0$ and some other polynomial $f$. It can be easily seen that if we multiply the integrand by $e^{-t}$ in each integral, then each integral will differ from the former one by the factor which belongs to $[1, e]$. So, this case follows from the previous one for $s=1$.

Remark 2.6. The estimate from Lemma 2.5 can not be extended to polynomials of the third degree. It is sufficient to take

$$
s=\infty, \quad f(t)=(t+1)^{2}(t-a) .
$$

Indeed, for $a>1$ and $\varepsilon<1$ we note that $f(t)<-a<-1<-\varepsilon$ for $t \in(0, a-1)$ and $f(t)>$ $(a+2)^{2}>1>\varepsilon$ for $t>a+1$. Thus, $\{f \leq-\varepsilon\} \supset(0, a-1),\{f \geq \varepsilon\} \supset(a+1,+\infty)$, and $\{|f| \leq \varepsilon\} \cap[0,+\infty) \subset(a-1, a+1)$. So,

$$
\begin{aligned}
\int_{0}^{+\infty} e^{-t} I_{\{f \leq-\varepsilon\}} d t \int_{0}^{+\infty} e^{-t} I_{\{f \geq \varepsilon\}} d t & \geq \int_{0}^{a-1} e^{-t} d t \int_{a+1}^{+\infty} e^{-t} d t=\left(1-e^{1-a}\right) e^{-a-1} \\
\int_{0}^{+\infty} e^{-t} I_{\{|f|<\varepsilon\}} d t & \leq \int_{a-1}^{a+1} e^{-t} I_{\left\{(t+1)^{2}|t-a|<\varepsilon\right\}} d t \\
& \leq \int_{a-1}^{a+1} e^{-t} I_{\left\{a^{2}|t-a|<\varepsilon\right\}} d t \leq e^{1-a} 2 \varepsilon a^{-2} \\
\int_{0}^{+\infty} e^{-t}(t+1)^{2}|t-a| d t & \leq\left(\int_{0}^{+\infty} e^{-t}(t+1)^{4} d t\right)^{1 / 2}\left(\int_{0}^{+\infty} e^{-t}(t-a)^{2} d t\right)^{1 / 2} \\
& =\left(\int_{0}^{+\infty} e^{-t}(t+1)^{4} d t\right)^{1 / 2}\left(1+(a-1)^{2}\right)^{1 / 2} \\
& \leq 2 a\left(\int_{0}^{+\infty} e^{-t}(t+1)^{4} d t\right)^{1 / 2}
\end{aligned}
$$

Thus, if estimate (2.5) is valid for such polynomials $f$ of the third degree with an absolute constant, then for another absolute constant $\tilde{c}$ one has

$$
\varepsilon\left(1-e^{1-a}\right) e^{-a-1} \leq \widetilde{c} e^{1-a} 2 \varepsilon a^{-2} a,
$$

which can not be true for $a$ large enough.

Remark 2.7. If the estimate from Lemma 2.5 were true for

$$
\int_{0}^{s} e^{-t}|f(t)-r| d t \quad \text { instead of } \int_{0}^{s} e^{-t}|f(t)| d t
$$


for every $r>0$, the isoperimetric inequality in the Cheeger form would be true for the distribution of any polynomial, see Lemma 3.1 and Theorem 3.5. However, this inequality cannot be true for an arbitrary log-concave measure. Indeed, this inequality for an arbitrary polynomial of degree two would have implied the exponential integrability of such polynomials with respect to an arbitrary log-concave measure (see [2]).

Lemma 2.8. There is an absolute constant c such that, for every polynomial of the second degree on $\mathbb{R}^{n}$, every log-concave measure $\mu$, and every positive number $\varepsilon$, the following inequality holds:

$$
\varepsilon \int I_{\{f \leq-\varepsilon\}} d \mu \int I_{\{f \geq \varepsilon\}} d \mu \leq c \int I_{\{|f|<\varepsilon\}} d \mu \int|f| d \mu .
$$

Proof. The functions $f_{1}=\varepsilon I_{\{f \leq-\varepsilon\}}, f_{2}=I_{\{f \geq \varepsilon\}}$ are upper semi-continuous and the functions $f_{3}=c I_{\{|f|<\varepsilon\}}$ and $f_{4}=|f|$ are lower semi-continuous. Hence, one can apply the Theorem 1.3. Thus, it is sufficient to prove the following inequality:

$$
\varepsilon \int_{s}^{r} e^{\ell(t)} I_{\{f \leq-\varepsilon\}} d t \int_{s}^{r} e^{\ell(t)} I_{\{f \geq \varepsilon\}} d t \leq c \int_{s}^{r} e^{\ell(t)} I_{\{|f|<\varepsilon\}} d t \int_{s}^{r} e^{\ell(t)}|f| d t,
$$

where $\ell$ is an affine function. By a linear change of variables, this estimate can be reduced to the estimate from Lemma 2.5. Hence, the theorem is proved.

Theorem 2.9. There is a constant $C>0$ such that for every polynomial of degree 2 on $\mathbb{R}^{n}$ and every log-concave measure $\mu$ the following estimate holds:

$$
\mu\left\{\left|f-m_{f}\right|<\varepsilon\right\} \int\left|f-m_{f}\right| d \mu \geq C \varepsilon \quad \text { for } \varepsilon<\alpha_{f} .
$$

Proof. This estimate follows from the previous theorem and Lemma 2.1.

\section{The isoperimetric and Poincaré inequalities}

The following lemma is an analog of Lemma 2.5 for polynomials of an arbitrary degree, but for measures with a density of the form $t^{n}$ on some interval instead of $e^{-t}$.

Lemma 3.1. For every pair of numbers $d, n \in \mathbb{N}$ there is a constant $c(d, n)$, depending only on $d$ and $n$, such that for every polynomial $f$ of degree $d$ on the real line and every pair of positive numbers $s, \varepsilon$ one has

$$
\varepsilon \int_{s}^{s+1} t^{n} I_{\{f \leq-\varepsilon\}} d t \int_{s}^{s+1} t^{n} I_{\{f \geq \varepsilon\}} d t \leq c(d, n) \int_{s}^{s+1} t^{n} I_{\{|f|<\varepsilon\}} d t \int_{s}^{s+1} t^{n}|f(t)| d t .
$$


Proof. Without loss of generality, we can assume that the coefficient at the highest degree term of the polynomial $f$ is 1 , that is, $f$ is of the form

$$
f(t)=\prod_{i=1}^{d}\left(t-t_{i}\right)
$$

Let

$$
\begin{aligned}
\mu_{s}(d t) & :=\left(\int_{s}^{s+1} t^{n} d t\right)^{-1} I_{[s, s+1]} t^{n} d t \\
m_{s} & :=\int t \mu_{s}(d t), \quad \sigma_{s}^{2}:=\int t^{2} \mu_{s}(d t)-m_{s}^{2} .
\end{aligned}
$$

Let

$$
c_{1}(n)=\inf _{s} \sigma_{s}^{2}
$$

Note that $c_{1}(n)>0$, since the limit at infinity of $\sigma_{s}^{2}$ is not zero. Let

$$
\tau:=\max \left\{t_{i}: t_{i} \in[s, s+1]\right\}
$$

Then on the interval $(\tau, s+1)$ the polynomial $f$ is either strictly positive or strictly negative. Hence,

$$
\int_{s}^{s+1} t^{n} I_{\{f \leq-\varepsilon\}} d t \int_{s}^{s+1} t^{n} I_{\{f \geq \varepsilon\}} d t \leq \int_{s}^{s+1} t^{n} d t \int_{s}^{\tau} t^{n} d t .
$$

Thus, it is sufficient to prove the estimate

$$
\varepsilon \int_{s}^{\tau} t^{n} d t \leq c(d, n) \int_{s}^{s+1} t^{n} I_{\{|f|<\varepsilon\}} d t \int|f| d \mu_{s} .
$$

Applying Theorem 1.1 to the polynomial $f$ and the measure $\mu_{s}$, we obtain

$$
\begin{aligned}
\|f\|_{L^{1}\left(\mu_{s}\right)}^{2} & \geq c^{-2 d}\|f\|_{L^{2}\left(\mu_{s}\right)}^{2} \geq c^{-2 d}\left\|f^{2}\right\|_{L^{0}\left(\mu_{s}\right)}=c^{-2 d} \prod_{i=1}^{d}\left\|\left(t-t_{i}\right)^{2}\right\|_{L^{0}\left(\mu_{s}\right)} \\
& \geq c^{-2 d}(2 c)^{-2 d} \prod_{i=1}^{d}\left\|\left(t-t_{i}\right)^{2}\right\|_{L^{1}\left(\mu_{s}\right)}=c^{-2 d}(2 c)^{-2 d} \prod_{i=1}^{d}\left(\sigma_{s}^{2}+\left|m_{s}-t_{i}\right|^{2}\right) .
\end{aligned}
$$

Let $t_{j}$ be a root of the polynomial $f$ such that $\alpha_{j}:=\inf _{t \in[s, s+1]}\left|t_{j}-t\right|>1$. Then

$$
|f(t)| \leq\left(\alpha_{j}+1\right) \prod_{i \neq j}\left|t-t_{i}\right|
$$


on $[s, s+1]$, while $\sigma_{s}^{2}+\left|m_{s}-t_{j}\right|^{2} \geq \alpha_{j}^{2}$. Let $t_{k}$ be a root of the polynomial $f$ such that $\alpha_{k}:=$ $\inf _{t \in[s, s+1]}\left|t_{k}-t\right| \leq 1$. Then $|f(t)| \leq 2 \prod_{i \neq k}\left|t-t_{i}\right|$ on $[s, s+1]$, while $\sigma_{s}^{2}+\left|m_{s}-t_{k}\right|^{2} \geq c_{1}(n)$. Applying these estimates several times, we obtain that

$$
|f(t)| \leq 2^{d}\left(\prod_{\alpha_{j}>1}\left(\alpha_{j}+1\right)\right)|t-\tau| \leq 4^{d}\left(\prod_{\alpha_{j}>1} \alpha_{j}\right)|t-\tau|
$$

on $[s, s+1]$, while

$$
\prod_{i=1}^{d}\left(\sigma_{s}^{2}+\left|m_{s}-t_{i}\right|^{2}\right) \geq\left(c_{1}(n)\right)^{d} \prod_{\alpha_{j}>1} \alpha_{j}^{2}
$$

Let $R:=\prod_{\alpha_{j}>1} \alpha_{j}$. Then it is sufficient to prove the estimate

$$
\varepsilon \int_{s}^{\tau} t^{n} d t \leq c(d, n) \int_{s}^{s+1} t^{n} I_{\left\{|t-\tau|<\varepsilon 4^{-d} R^{-1}\right\}} d t \int|f| d \mu_{s} .
$$

First, we consider the case $\varepsilon<2 \int|f| d \mu_{s}$. If $\varepsilon 4^{-d} R^{-1}>1 / 4$, then

$$
\begin{gathered}
\varepsilon \int_{s}^{\tau} t^{n} d t \leq 2 \int|f| d \mu_{s}(n+1)^{-1}\left((s+1)^{n+1}-s^{n+1}\right), \\
\int_{s}^{s+1} t^{n} I_{\left\{|t-\tau|<\varepsilon 4^{-d} R^{-1}\right\}} d t \int|f| d \mu_{s} \geq(n+1)^{-1}\left((s+1 / 4)^{n+1}-s^{n+1}\right) \int|f| d \mu_{s} .
\end{gathered}
$$

Obviously, $\inf _{s}\left((s+1 / 4)^{n+1}-s^{n+1}\right)\left((s+1)^{n+1}-s^{n+1}\right)^{-1}>0$, thus, in that case the desired inequality is proved.

Let now $\varepsilon 4^{-d} R^{-1} \leq 1 / 4$. It is sufficient to prove the inequality

$$
\varepsilon \int_{s}^{\tau} t^{n} d t \leq c(d, n) R \int_{s}^{s+1} t^{n} I_{\left\{|t-\tau|<\varepsilon 4^{-d} R^{-1}\right\}} d t,
$$

since $\int|f| d \mu_{s} \geq \delta(d, n) R$. If $\tau<s+3 / 4$, then

$$
\int_{s}^{s+1} t^{n} I_{\left\{|t-\tau|<\varepsilon 4^{-d} R^{-1}\right\}} d t \geq \tau^{n} \varepsilon 4^{-d} R^{-1},
$$

since $\left[\tau, \tau+\varepsilon 4^{-d} R^{-1}\right] \subset[s, s+1]$, while

$$
\int_{s}^{\tau} t^{n} d t \leq \tau^{n}(\tau-s) \leq \tau^{n}
$$

Thus, in this case the desired inequality is also proved. Let now $\tau \geq s+3 / 4$. Then

$$
\int_{s}^{s+1} t^{n} I_{\left\{|t-\tau|<\varepsilon 4^{-d} R^{-1}\right\}} d t \geq\left(\tau-\varepsilon 4^{-d} R^{-1}\right)^{n} \varepsilon 4^{-d} R^{-1},
$$


since $\left[\tau-\varepsilon 4^{-d} R^{-1}, \tau\right] \subset[s, s+1]$. The last expression can be estimated by

$$
(\tau-1 / 4)^{n} \varepsilon 4^{-d} R^{-1} \geq(s+1 / 2)^{n} \varepsilon 4^{-d} R^{-1} .
$$

Moreover,

$$
\int_{s}^{\tau} t^{n} d t \leq(s+1)^{n}
$$

and, since $s+1 / 2 \geq 1 / 2(s+1)$, the desired inequality is proved in this case too.

It remains to consider the case where $\varepsilon \geq 2 \int|f| d \mu_{s}$. Applying Chebyshev's inequality, we obtain

$$
\mu_{s}(|f| \geq \varepsilon) \leq \varepsilon^{-1} \int|f| d \mu_{s} \leq 1 / 2
$$

hence

$$
\mu_{S}(|f|<\varepsilon) \geq 1-1 / 2=1 / 2 .
$$

Thus, we have

$$
\varepsilon \mu_{s}\{f \leq-\varepsilon\} \mu_{s}\{f \geq \varepsilon\} \leq c(d, n) \mu_{s}\{|f|<\varepsilon\} \int|f| d \mu_{s},
$$

which is equivalent to the announced estimate.

For the further purposes, we need the following lemma, which is nontrivial, since the constant in the estimate of $L^{2}$-norm of a polynomial in terms of $L^{2}$-norm of its derivative depends only on the degree of the polynomial and on the variance of the measure.

Lemma 3.2 (The reverse Poincaré inequality). Let $d \in \mathbb{N}$. Then there is an absolute constant $C$, independent of measure $\mu$, such that for every polynomial $f$ of degree $d$ on the real line and for every log-concave measure $\mu$ on the real line the following inequality holds:

$$
\sigma(\mu)\left\|f^{\prime}\right\|_{L^{2}(\mu)} \leq(C d)^{d+1}\|f\|_{L^{2}(\mu)},
$$

where $\sigma^{2}(\mu)$ is the variance of the measure $\mu$.

Proof. Due to homogeneity, we can assume that the polynomial $f$ is of the form

$$
f(t)=\prod_{i=1}^{d}\left(t-t_{i}\right)
$$

Also, without loss of generality we can assume that $\int t \mu(d t)=0$. Applying the estimate of the $L^{1}$-norm of a polynomial via its $L^{0}$-norm from Theorem 1.1, using multiplicativity of the 
$L^{0}$-norm and estimating the $L^{0}$-norm by the $L^{1}$-norm, we obtain

$$
\begin{aligned}
\int t^{2} \mu(d t) \int\left(f^{\prime}(t)\right)^{2} \mu(d t) & \leq d \sum_{i=1}^{d} \int t^{2} \mu(d t) \int\left|\prod_{j \neq i}\left(t-t_{j}\right)\right|^{2} \mu(d t) \\
& \leq d(2 c d)^{2 d} \sum_{i=1}^{d} \int t^{2} \mu(d t) \prod_{j \neq i} \int\left|t-t_{j}\right|^{2} \mu(d t) \\
& =d(2 c d)^{2 d} \sum_{i=1}^{d} \int t^{2} \mu(d t) \prod_{j \neq i}\left(\int t^{2} \mu(d t)+\left|t_{j}\right|^{2}\right) \\
& \leq d^{2}(2 c d)^{2 d} \prod_{i=1}^{d}\left(\int t^{2} \mu(d t)+\left|t_{i}\right|^{2}\right) \\
& =d^{2}(2 c d)^{2 d} \prod_{i=1}^{d} \int\left|t-t_{i}\right|^{2} \mu(d t) \leq d^{2}\left(4 c^{2} d\right)^{2 d} \int f^{2} \mu(d t),
\end{aligned}
$$

as announced.

Lemma 3.3. Let $d, n \in \mathbb{N}$. Then there is a constant $C(d, n)$, which depends only on $d$ and $n$, such that, for every $s \geq 0$, every polynomial $f$ of degree $d$ on $\mathbb{R}$, which vanishes at some point $\tau \in[s, s+1]$, and every real number $r$, one has

$$
\int_{s}^{s+1}|f(t)| t^{n} d t \leq C(d, n) \int_{s}^{s+1}|f(t)-r| t^{n} d t .
$$

Proof. Let $\mu_{s}, \sigma_{s}$ be defined as in the proof of Lemma 3.1. Note that it is sufficient to prove the inequality

$$
\int|f| d \mu_{s} \leq C(d, n) \int|f-r| d \mu_{s}
$$

It can be easily verified that

$$
\|f\|_{L^{\infty}\left(\mu_{s}\right)} \leq\left\|f^{\prime}\right\|_{L^{\infty}\left(\mu_{s}\right)},
$$

since $f(\tau)=0$ for some point $\tau \in[s, s+1]$. Recall the following inequality (see [17], Theorem 3 ) that holds with some universal constant $C$ for any probability measure on some interval with a density of the form $c t^{n}$ and for any polynomial $g$ of degree $d$ :

$$
\|g\|_{\infty} \leq\left(C \max \left\{1, \frac{n+1}{d}\right\}\right)^{d}\|g\|_{2} .
$$


Applying this estimate to our measure $\mu_{s}$ and the polynomial $f^{\prime}$, we obtain

$$
\left\|f^{\prime}\right\|_{L^{\infty}\left(\mu_{s}\right)} \leq\left(C \max \left\{1, \frac{n+1}{d-1}\right\}\right)^{d-1}\left\|f^{\prime}\right\|_{L^{2}\left(\mu_{s}\right)} .
$$

We now use Lemma 3.2:

$$
\left\|f^{\prime}\right\|_{L^{2}\left(\mu_{s}\right)} \leq(C d)^{d+1}\|f-r\|_{L^{2}(\mu)} \sigma_{s}^{-1} .
$$

Since $\inf _{s} \sigma_{s}>0$ and $\|f\|_{L^{1}\left(\mu_{s}\right)} \leq\|f\|_{L^{\infty}\left(\mu_{s}\right)}$, the lemma is proved.

Corollary 3.4. Let $n, d \in \mathbb{N}$. Then there is a constant $c(d, n)$ depending only on $d$ and $n$ such that for every polynomial $f$ of degree $d$ on the real line, every pair of positive numbers $s, \varepsilon$ and every real number $r$ one has

$$
\varepsilon \int_{s}^{s+1} t^{n} I_{\{f \leq-\varepsilon\}} d t \int_{s}^{s+1} t^{n} I_{\{f \geq \varepsilon\}} d t \leq c(d, n) \int_{s}^{s+1} t^{n} I_{\{|f|<\varepsilon\}} d t \int_{s}^{s+1} t^{n}|f(t)-r| d t .
$$

Proof. If $f$ has no zeros in $[s, s+1]$, then the left-hand side vanishes and the inequality is obvious. If $f$ has a root in $[s, s+1]$, then we can apply Lemmas 3.1 and 3.3.

Theorem 3.5. Let $K$ be a convex compact set in $\mathbb{R}^{n}$, let $\lambda_{K}$ be the normalized Lebesgue measure on $K$, and let $f$ be a polynomial of degree $d$. Let $\mathbb{R}=J_{1} \sqcup J_{2} \sqcup J_{3}$, where $J_{i}$ are disjoint measurable sets, and let the distance between $J_{1}$ and $J_{3}$ be $\varepsilon>0$, i.e.,

$$
\inf _{x_{1} \in J_{1}, x_{2} \in J_{3}}\left|x_{1}-x_{2}\right|=\varepsilon>0 .
$$

Then there is a constant $c(d, n)$ depending only on $d$ and $n$ such that

$$
\varepsilon \lambda_{K}\left(f \in J_{1}\right) \lambda_{K}\left(f \in J_{3}\right) \leq c(d, n) \lambda_{K}\left(f \in J_{2}\right) \int_{K}\left|f-m_{f}\right| d \lambda_{K},
$$

where $m_{f}=\int f d \lambda_{K}$.

Proof. Obviously, it is sufficient to prove the estimate with an arbitrary number $m$ in place of $m_{f}$. We will prove exactly this statement.

If we take the closures of the sets $J_{1}$ and $J_{3}$ and replace $J_{2}$ with $\mathbb{R} \backslash\left(J_{1} \cup J_{3}\right)$, the left-hand side of the inequality does not decrease, while the right-hand side does not increase. Hence we can assume that $J_{1}$ and $J_{3}$ are closed and $J_{2}$ is open. First we consider the case where $J_{2}$ is an interval. Note that our inequality can be written in the form

$$
\varepsilon \int_{K} I_{\left\{f \in J_{1}\right\}}(x) d x \int_{K} I_{\left\{f \in J_{3}\right\}}(x) d x \leq c(d, n) \int_{K} I_{\left\{f \in J_{2}\right\}}(x) d x \int_{K}|f(x)-m| d x .
$$

Since $J_{1}$ and $J_{3}$ are closed and $J_{2}$ is open, the functions $f_{1}=\varepsilon I_{\left\{f \in J_{1}\right\}}, f_{2}=I_{\left\{f \in J_{3}\right\}}$ are upper semi-continuous and the functions $f_{3}=c(d, n) I_{\left\{f \in J_{2}\right\}}, f_{4}=|f-m|$ are lower semi-continuous. 
Hence, we can apply Theorem 1.4. Thus, it is sufficient to prove the inequality

$$
\begin{aligned}
& \varepsilon \int_{s}^{r} \ell(t)^{n-1} I_{\left\{f \in J_{1}\right\}} d t \int_{s}^{r} \ell(t)^{n-1} I_{\left\{f \in J_{3}\right\}} d t \\
& \quad \leq c(d, n) \int_{s}^{r} \ell(t)^{n-1} I_{\left\{f \in J_{2}\right\}} d t \int_{s}^{r} \ell(t)^{n-1}|f-m| d t,
\end{aligned}
$$

where $\ell$ is an affine function that is nonnegative on $[s, r]$. By a linear change of variables, we arrive at the inequality

$\varepsilon \int_{s}^{s+1} t^{n-1} I_{\left\{f \in J_{1}\right\}} d t \int_{s}^{s+1} t^{n-1} I_{\left\{f \in J_{3}\right\}} d t \leq c(d, n) \int_{s}^{s+1} t^{n-1} I_{\left\{f \in J_{2}\right\}} d t \int_{s}^{s+1} t^{n-1}|f-m| d t$,

where $s \geq 0$ and $f$ is some (possibly, different) polynomial of the same degree. Thus, the case where $J_{2}$ is an open interval follows from Corollary 3.4.

Recall that $\mu_{f}=\lambda_{K} \circ f^{-1}$ is the image of the uniform distribution on the compact set $K$ under the polynomial mapping $f$. Note that the support of the measure $\mu_{f}$ is a bounded set on the real line. Let now $J_{2}$ be the union of countably many disjoint intervals. The proof in this case is similar to the one from [22]. For completeness, we present this argument. If there is an interval which length is less than $\varepsilon$, then both its end points belong either to $J_{1}$ or to $J_{3}$ and we can add this interval to $J_{1}$ or $J_{3}$, respectively, and prove the estimate for exactly these sets. Thus, we assume that $J_{2}=\bigsqcup_{i}\left(a_{i}, b_{i}\right)$ (the union of disjoint intervals), moreover, $b_{i}-a_{i} \geq \varepsilon$ and there are finitely many such intervals, since the support of the measure $\mu_{f}$ is bounded. We have already proved that

$$
\varepsilon \mu_{f}\left(\left(-\infty, a_{i}\right]\right) \mu_{f}\left(\left[b_{i}, \infty\right)\right) \leq c(d, n) \mu_{f}\left(\left(a_{i}, b_{i}\right)\right) \alpha_{f} .
$$

Summing these inequalities in $i$, we obtain

$$
\sum_{i} \mu_{f}\left(\left(-\infty, a_{i}\right]\right) \mu_{f}\left(\left[b_{i}, \infty\right)\right) \leq \frac{c(d, n) \alpha_{f}}{\varepsilon} \mu_{f}\left(J_{2}\right) .
$$

Since every point of $J_{1}$ and every point of $J_{3}$ are separated by at least one interval $\left(a_{i}, b_{i}\right)$, we have

$$
\sum_{i} \mu_{f}\left(\left(-\infty, a_{i}\right]\right) \mu_{f}\left(\left[b_{i}, \infty\right)\right) \geq \mu_{f}\left(J_{1}\right) \mu_{f}\left(J_{3}\right),
$$

which completes the proof.

Corollary 3.6 (The isoperimetric inequality in Cheeger's form). For every numbers $n, d \in \mathbb{N}$ there is a constant $\delta(d, n)$ depending only on $d$ and $n$ such that for every convex compact set $K$ in $\mathbb{R}^{n}$ and for every polynomial $f$ of degree $d$ one has

$$
\mu_{f}^{+}(A) \geq \frac{\delta(d, n)}{\alpha_{f}} \mu_{f}(A) \mu_{f}(\mathbb{R} \backslash A),
$$

where $\mu_{f}:=\lambda_{K} \circ f^{-1}$ and $\lambda_{K}$ is the normalized Lebesgue measure on $K$. 
Proof. It is sufficient to apply the previous theorem to the sets

$$
J_{1}=A, \quad J_{3}=\mathbb{R} \backslash(A+(-\varepsilon, \varepsilon)), \quad J_{2}=\mathbb{R} \backslash\left(J_{1} \cup J_{2}\right)
$$

and let $\varepsilon$ tend to zero.

As it is known (see [18], a proof can also be found in [2]), the previous assertion implies the following result.

Corollary 3.7 (The Poincaré inequality). For every numbers $n, d \in \mathbb{N}$ there is a constant $C(d, n)$ depending only on $d$ and $n$ such that for every convex compact set $K$ in $\mathbb{R}^{n}$, for every polynomial $f$ of degree $d$ and for every smooth function $\varphi$ one has

$$
\left\|\varphi-\int \varphi d \mu_{f}\right\|_{L^{2}\left(\mu_{f}\right)} \leq C(d, n) \alpha_{f}\left\|\varphi^{\prime}\right\|_{L^{2}\left(\mu_{f}\right)},
$$

where $\mu_{f}:=\lambda_{K} \circ f^{-1}$ and $\lambda_{K}$ is the normalized Lebesgue measure on $K$.

\section{Acknowledgements}

We would like to thank the referees for the simplification of the proof of Lemma 2.1 and for various helpful comments and suggestions which have significantly improved the presentation of our paper.

This work has been supported by the Russian Science Foundation Grant 14-11-00196 at Lomonosov Moscow State University.

\section{References}

[1] Arutyunyan, L.M. and Kosov, E.D. (2015). Estimates for integral norms of polynomials on spaces with convex measures. Mat. Sb. 206 3-22. MR3438588

[2] Bobkov, S.G. (1997). Isoperimetric problems in the theory of infinite dimensional probability distributions. Doctoral dissertation, Saint Petersburg.

[3] Bobkov, S.G. (1999). Isoperimetric and analytic inequalities for log-concave probability measures. Ann. Probab. 27 1903-1921. MR1742893

[4] Bobkov, S.G. (2000). Remarks on the growth of $L^{p}$-norms of polynomials. In Geometric Aspects of Functional Analysis. Lecture Notes in Math. 1745 27-35. Berlin: Springer. MR1796711

[5] Bobkov, S.G. (2000). Some generalizations of Prokhorov's results on Khinchin-type inequalities for polynomials. Theory Probab. Appl. 45 644-647. MR1968725

[6] Bobkov, S.G. (2003). Spectral gap and concentration for some spherically symmetric probability measures. In Geometric Aspects of Functional Analysis. Lecture Notes in Math. 1807 37-43. Berlin: Springer. MR2083386

[7] Bobkov, S.G. (2009). On the isoperimetric constants for product measures. J. Math. Sci. (N. Y.) 159 47-53. MR2544042 
[8] Bobkov, S.G. and Houdré, C. (1997). Isoperimetric constants for product probability measures. Ann. Probab. 25 184-205. MR1428505

[9] Bogachev, V.I. (1998). Gaussian Measures. Mathematical Surveys and Monographs 62. Providence, RI: Amer. Math. Soc. MR1642391

[10] Bogachev, V.I. (2010). Differentiable Measures and the Malliavin Calculus. Mathematical Surveys and Monographs 164. Providence, RI: Amer. Math. Soc. MR2663405

[11] Bogachev, V.I., Kosov, E.D., Nourdin, I. and Poly, G. (2015). Two properties of vectors of quadratic forms in Gaussian random variables. Theory Probab. Appl. 59 208-221. MR3416047

[12] Bogachev, V.I. and Zelenov, G.I. (2015). On convergence in variation of weakly convergent multidimensional distributions. Dokl. Akad. Nauk 461 14-17. MR3442783

[13] Borell, C. (1974). Convex measures on locally convex spaces. Ark. Mat. 12 239-252. MR0388475

[14] Borell, C. (1975). Convex set functions in $d$-space. Period. Math. Hungar. 6 111-136. MR0404559

[15] Borell, C. (1975). The Brunn-Minkowski inequality in Gauss space. Invent. Math. 30 207-216. MR0399402

[16] Bourgain, J. (1991). On the distribution of polynomials on high-dimensional convex sets. In Geometric Aspects of Functional Analysis (1989-1990). Lecture Notes in Math. 1469 127-137. Berlin: Springer. MR1122617

[17] Carbery, A. and Wright, J. (2001). Distributional and $L^{q}$ norm inequalities for polynomials over convex bodies in $\mathbb{R}^{n}$. Math. Res. Lett. 8 233-248. MR1839474

[18] Cheeger, J. (1970). A lower bound for the smallest eigenvalue of the Laplacian. In Problems in Analysis (Papers Dedicated to Salomon Bochner, 1969) 195-199. Princeton, NJ: Princeton Univ. Press. MR0402831

[19] Fradelizi, M. and Guédon, O. (2004). The extreme points of subsets of $s$-concave probabilities and a geometric localization theorem. Discrete Comput. Geom. 31 327-335. MR2060645

[20] Friedland, O. and Sodin, S. (2007). Bounds on the concentration function in terms of the Diophantine approximation. C. R. Math. Acad. Sci. Paris 345 513-518. MR2375113

[21] Gromov, M. and Milman, V.D. (1987). Generalization of the spherical isoperimetric inequality to uniformly convex Banach spaces. Compos. Math. 62 263-282. MR0901393

[22] Kannan, R., Lovász, L. and Simonovits, M. (1995). Isoperimetric problems for convex bodies and a localization lemma. Discrete Comput. Geom. 13 541-559. MR1318794

[23] Klartag, B. (2006). On convex perturbations with a bounded isotropic constant. Geom. Funct. Anal. 16 1274-1290. MR2276540

[24] Littlewood, J.E. and Offord, A.C. (1943). On the number of real roots of a random algebraic equation. III. Rec. Math. [Mat. Sbornik] N.S. 12(54) 277-286. MR0009656

[25] Lovász, L. and Simonovits, M. (1993). Random walks in a convex body and an improved volume algorithm. Random Structures Algorithms 4 359-412. MR1238906

[26] Nazarov, F., Sodin, M. and Volberg, A. (2002). The geometric Kannan-Lovász-Simonovits lemma, dimension-free estimates for the distribution of the values of polynomials, and the distribution of the zeros of random analytic functions. Algebra i Analiz 14 214-234. MR1925887

[27] Nourdin, I., Nualart, D. and Poly, G. (2013). Absolute continuity and convergence of densities for random vectors on Wiener chaos. Electron. J. Probab. 18 no. 22, 19. MR3035750

[28] Nourdin, I. and Poly, G. (2013). Convergence in total variation on Wiener chaos. Stochastic Process. Appl. 123 651-674. MR3003367

[29] Rudelson, M. and Vershynin, R. (2009). Smallest singular value of a random rectangular matrix. Comm. Pure Appl. Math. 62 1707-1739. MR2569075

[30] Rudelson, M. and Vershynin, R. (2015). Small ball probabilities for linear images of high-dimensional distributions. Int. Math. Res. Not. IMRN 19 9594-9617. MR3431603 
[31] Sudakov, V.N. and Cirel'son, B.S. (1974). Extremal properties of half-spaces for spherically invariant measures. Zap. Nauchn. Sem. S.-Peterburg. Otdel. Mat. Inst. Steklov. (POMI) 41 14-24, 165. MR0365680

Received April 2016 and revised November 2016 